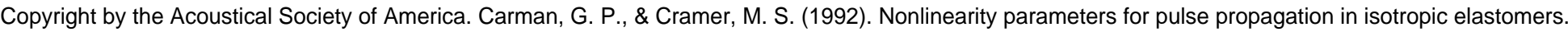
Journal of the Acoustical Society of America, 91(1), 39-51. doi: 10.1121/1.402727

\title{
Nonlinearity parameters for pulse propagation in isotropic elastomers
}

\author{
G. P. Carman and M. S. Cramer \\ Engineering Science and Mechanics, Virginia Polytechnic Institute and State University, Blacksburg, Virginia \\ 24061
}

(Received 8 March 1991; accepted for publication 30 August 1991)

The propagation of one-dimensional shear waves in isotropic hyperelastic materials is examined. Both compressible and incompressible materials subject to arbitrarily large shear prestrains are considered. The nonlinear evolution equation governing small disturbances on prestrained undisturbed states is derived. The prestrain is seen to change the nonlinearity from the cubic form found in the unstrained case to the stronger quadratic form governed by the conventional Burgers' equation. Additional results include explicit and general expressions for the quadratic and cubic nonlinearity parameters. Numerical estimates are also provided for natural rubber and foamed polyurethane. It is demonstrated that the quadratic nonlinearity parameter does not increase monotonically with prestrain and may actually vanish at nonzero values of the prestrain.

PACS numbers: $43.25 . \mathrm{Ba}$

\section{INTRODUCTION}

The dynamics of elastomers are of considerable scientific and practical interest in many areas of acoustics and classical mechanics. The practical interest stems in part from their use as shock and vibration isolators, automobile tires being the most conspicuous example, and sonar applications. From a scientific point of view, their ability to sustain large elastic deformations permits testing of elasticity models well beyond the range explored for metals and ceramics.

When such large deformations occur, the behavior of these materials will differ significantly from that predicted by the well-known theory of linear elasticity. This is due to the fact that nonlinearities in the material response must be considered. Such nonlinearities inevitably lead to harmonic generation and shock formation. A detailed analysis of the former in longitudinal waves has been provided by Thurston and Shapiro (1967). The reader is also referred to the experimental studies of R. B. Thompson and co-workers (1976, 1977) and Peters and Breazeale (1968). Studies of nonlinear shear waves have been carried out by Chu (1964) and Davison (1966). These latter studies employed the exact method of characteristics to examine the wave families possible in incompressible and compressible materials, respectively.

A more detailed study of the distortion of shear waves is presented by Lee-Bapty (1981) who demonstrated that the basic wave evolution is fundamentally different than that exhibited by longitudinal waves. The nonlinearity of relatively weak longitudinal waves is quadratic leading to the generation of second harmonics when wave trains are considered, whereas the nonlinearity of shear waves in an initially unstrained material is seen to be cubic. Because of the cubic nonlinearity, second harmonics are suppressed in wave trains and even the simplest pulses tend to evolve into asymptotic profiles containing two shocks, rather than one as in the case of quadratic nonlinearity. It is also useful to note that this cubic nonlinearity is an order of magnitude weaker than the quadratic nonlinearity. For example, the shock formation time is on the order of the inverse square of the wave amplitude when the nonlinearity is cubic as compared to inversely proportional when the nonlinearity is quadratic. A more complete discussion of these nonclassical dynamics may be found in the thesis by Lee-Bapty (1981) or the article by Lee-Bapty and Crighton (1987).

In the present study, we examine the one-dimensional evolution of small disturbances propagating in elastomers having an arbitrarily large shear prestrain. Both incompressible and compressible materials are considered. The present study extends the work of Nariboli and Lin (1973) and LeeBapty (1981) who considered only unstrained materials. We apply the well-known reductive perturbation technique of Taniuti and Wei (1968) to derive the nonlinear evolution equation governing small shear disturbances over time scales on the order of the shock formation time. General expressions for the quadratic steepening parameter (the ana$\log$ of $1+B / 2 A$ in the theory of compressible fluids) are given and it is shown that this parameter is generally nonzero for arbitrary prestrain values. That is, prestraining the material causes the nonlinearity to change from the cubic nonlinearity to the quadratic nonlinearity analogous to that encountered in studies of the longitudinal mode. This not only increases the strength of the nonlinearity but also modifies the details of the evolution.

It is of interest to note that such a cubic-quadratic transition also occurs in the study of non-Newtonian fluids. A summary of this effect in the context of harmonic generation has been provided by Powell and Schwarz (1979).

The numerical examples provided in Secs. V and VI reveal that the quadratic nonlinearity parameter tends to be negative at the lower values of prestrain, at least for the materials considered here. Thus the wave evolution is characterized by a backward steepening and is similar to that found by Bains and Breazeale (1975) in their study of longitudinal waves in fused silica and the well-known phenomenon asso- 
ciated with temperature waves in superfluid helium, see, e.g., Putterman (1974). The numerical estimates provided here also suggest that this quadratic nonlinearity parameter vanishes at larger values of the prestrain again resulting in a cubic nonlinearity. At even larger prestrains, the steepening is forward and resembles that of longitudinal waves in many solids and dilute gases. Thus the behavior of nonlinear shear waves more closely resembles that of other areas of nonlinear acoustics in that the quadratic nonlinearity parameter is generally nonzero and changes sign, i.e., vanishes, only at discrete undisturbed states.

Throughout we will refer to the points at which the quadratic steepening parameter vanishes as critical points. As indicated above, the nonlinearity at these points is cubic rather than quadratic and the usual Burgers' equation does not give an accurate representation of the nonlinear evolution. As pointed out by Nariboli and Lin (1973) and LeeBapty (1981), the wave distortion occurs more slowly and longer time scales must be employed. In Sec. III, we apply an extension of the perturbation technique of Taniuti and Wei (1968), as developed by Cramer and Sen (1991), to derive the evolution equation governing the nonlinear distortion not only at, but in the neighborhood of, each critical point. The resultant evolution equation is a modification of Burgers' equation containing both quadratic and cubic nonlinearity. At critical points this equation reduces, as it should, to the cubic equation derived by previous investigators. In addition to providing general expressions for the cubic nonlinearity parameter, we also give numerical estimates based on specific material models.

\section{FORMULATION}

The general relation for a hyperelastic isotropic solid undergoing isothermal deformation (Hunter, 1983) may be written as follows:

$$
\sigma_{i j}=\alpha_{0} \delta_{i j}+\alpha_{1} B_{i j}+\alpha_{2} B_{i r} B_{r j},
$$

where $\sigma_{i j}$ is the Cauchy stress tensor, $\delta_{i j}$ is the Kronecker delta, and $B_{i j}$ is the left Cauchy-Green tensor. It should be pointed out that in all equations throughout this paper, unless otherwise noted, the standard range and summation convention applies to all subscripted lower case Latin indices in three space. The left Cauchy-Green tensor is related to the deformation gradient $F_{i j}$ by

$$
B_{i j}=F_{i r} F_{j r} \text {. }
$$

The deformation gradient is defined as the gradient of the instantaneous material position $x_{j}$ (i.e., Eulerian) with respect to the initial material position $X_{j}$ (i.e., Lagrangian). That is,

$$
F_{i j}=\frac{\partial x_{i}}{\partial X_{j}} .
$$

The displacement vector $u_{i}$ is defined as follows:

$$
x_{j}=X_{j}+u_{j}\left(X_{1} ; X_{2}, X_{3}, t\right) \text {. }
$$

The scalar quantities $\alpha_{0}, \alpha_{1}$, and $\alpha_{2}$ in Eq. (1) have as yet to be defined. These terms are related to the free energy $U$ of the solid and the three invariants $\left(\mathrm{I}_{B}, \mathrm{II}_{B}\right.$, and $\left.\mathrm{III}_{B}\right)$ of the left Cauchy-Green tensor which are written as follows:

$$
\mathrm{I}_{B} \equiv B_{r r}, \quad \mathrm{II}_{B} \equiv \frac{1}{2}\left(\mathrm{I}_{B}^{2}-B_{k l} B_{l k}\right), \quad \mathrm{III}_{B} \equiv \operatorname{det}\left(B_{i j}\right),
$$

through the following equations (Hunter, 1983):

$$
\begin{aligned}
& \alpha_{0}=2 \rho \mathrm{III}_{B} \frac{\partial U}{\partial \mathrm{III}_{B}}, \quad \alpha_{1}=2 \rho\left(\frac{\partial U}{\partial \mathrm{I}_{B}}+\mathrm{I}_{B} \frac{\partial U}{\partial \mathrm{II}_{B}}\right), \\
& \alpha_{2}=-2 \rho \frac{\partial U}{\partial \mathrm{II}_{B}} .
\end{aligned}
$$

The quantity $\rho\left(X_{i}, t\right)$ is defined to be the density distribution in the solid at an arbitrary time $t$. The density can be related to some initial density of the solid $\rho_{0}$ at time $t_{0}$ and the determinant of the left Cauchy-Green tensor by

$$
\rho=\rho_{0} \mathrm{III}_{B}^{-1 / 2} \text {. }
$$

The momentum equations, neglecting body forces, may be expressed as

$$
\rho \frac{\partial^{2} u_{i}}{\partial t^{2}}=\frac{\partial \sigma_{j i}}{\partial x_{j}} .
$$

The remainder of this section will specifically address the formulation of the equations describing a shear pulse introduced into a hyperelastic solid. It is well known that a shear pulse propagating in a nonlinear compressible solid generates a longitudinal compressive wave of higher order (Davison, 1966). Thus the simplest displacement field is of the form

$x_{1}=X_{1}+u_{1}\left(X_{1}, t\right), \quad x_{2}=X_{2}, \quad x_{3}=X_{3}+u_{3}\left(X_{1}, t\right)$.

The $B_{i j}$ corresponding to the deformation field (9) may therefore be represented

$$
\left[\begin{array}{ccc}
\left(1+e_{1}\right)^{2} & 0 & \gamma_{5}\left(1+e_{1}\right) \\
0 & 1 & 0 \\
\gamma_{5}\left(1+e_{1}\right) & 0 & \gamma_{5}^{2}+1
\end{array}\right] .
$$

In the expression (10) and subsequent equations, the ensuing relations between the linear normal $\left(e_{1}\right)$ and shear $\left(\gamma_{5}\right)$ strains and the gradients of $u_{i}$ have been used:

$$
e_{1}=\frac{\partial u_{1}}{\partial X_{1}} \quad \text { and } \gamma_{5}=\frac{\partial u_{3}}{\partial X_{1}} \text {. }
$$

The invariants defined in Eq. (5) are thus found to be

$$
\begin{array}{ll}
\mathrm{I}_{B}=3+2 e_{1}+e_{1}^{2}+\gamma_{5}^{2}, & \mathrm{II}_{B}=3+4 e_{1}+2 e_{1}^{2}+\gamma_{5}^{2}, \\
\mathrm{III}_{B}=1+2 e_{1}+e_{1}^{2}, & \text { (12) }
\end{array}
$$

and the nonzero components of the stress tensor can be written

$$
\begin{aligned}
\sigma_{1}= & \alpha_{0}+\alpha_{1}\left(1+e_{1}\right)^{2}+\alpha_{2}\left[\left(1+e_{1}\right)^{4}+\gamma_{5}^{2}\left(1+e_{1}\right)^{2}\right], \\
\sigma_{2}= & \alpha_{0}+\alpha_{1}+\alpha_{2}, \\
\sigma_{3}= & \alpha_{0}+\alpha_{1}\left(1+\gamma_{5}^{2}\right)+\alpha_{2}\left[\gamma_{5}^{2}\left(1+e_{1}\right)^{2}+\left(1+\gamma_{5}^{2}\right)^{2}\right], \\
\sigma_{5}= & \alpha_{1} \gamma_{5}\left(1+e_{1}\right) \\
& +\alpha_{2}\left[\gamma_{5}\left(1+e_{1}\right)^{3}+\left(\gamma_{5}^{3}+\gamma_{5}\right)\left(1+e_{1}\right)\right] .
\end{aligned}
$$

Here $\sigma_{1} \equiv \sigma_{11}, \sigma_{2} \equiv \sigma_{22}, \sigma_{3} \equiv \sigma_{33}$, and $\sigma_{5} \equiv \sigma_{31}$. By using Eqs. (1)-(3) and (5)-(9) the following relationships can be derived between Cauchy stress, linear strain, and displacement: 


$$
\begin{aligned}
& \frac{\partial \sigma_{1}}{\partial e_{1}} \frac{\partial e_{1}}{\partial X_{1}}+\frac{\partial \sigma_{1}}{\partial \gamma_{5}} \frac{\partial \gamma_{5}}{\partial X_{1}}=\rho_{0} \frac{\partial^{2} u_{1}}{\partial t^{2}}, \\
& \frac{\partial \sigma_{5}}{\partial e_{1}} \frac{\partial e_{1}}{\partial X_{1}}+\frac{\partial \sigma_{5}}{\partial \gamma_{5}} \frac{\partial \gamma_{5}}{\partial X_{1}}=\rho_{0} \frac{\partial^{2} u_{3}}{\partial t^{2}} .
\end{aligned}
$$

For reasons which will become evident, it is desirable to recast Eq. (14) in the following nondimensional form:

$$
\frac{\partial w_{\zeta}}{\partial \bar{t}}+A_{\zeta \eta}\left(w_{\xi}\right) \frac{\partial w_{\eta}}{\partial \bar{X}_{1}}=0 .
$$

Subscripted Greek indices in Eq. (15) and in subsequent equations are ranged and summed over a four space. The solution vector $w_{\eta}$ is comprised of the linear strains and the nondimensional velocities $\bar{v}_{1}$ and $\bar{v}_{3}$ (the dimensional velocities are $v_{1}=\partial u_{1} / \partial t$ and $\left.v_{3}=\partial u_{3} / \partial t\right)$ and can be written

$$
\left[\begin{array}{l}
\bar{v}_{1} \\
\bar{v}_{3} \\
e_{1} \\
\gamma_{5}
\end{array}\right]
$$

The quantity $A_{\zeta \eta}$ is a $4 \times 4$ matrix defined as follows:

$$
\left[\begin{array}{cccc}
0 & 0 & -\sigma_{1,1} / \rho_{0} C_{s}^{2} & -\sigma_{1,5} / \rho_{0} C_{s}^{2} \\
0 & 0 & -\sigma_{5,1} / \rho_{0} C_{s}^{2} & -\sigma_{5,5} / \rho_{0} C_{s}^{2} \\
-1 & 0 & 0 & 0 \\
0 & -1 & 0 & 0
\end{array}\right] .
$$

We refer to (17) as the speed matrix even though the stress gradients may generally vary with the local strain. It should be noted that commas on the stress tensor denote partial differentiation with respect to the linear strain variables. That is $f_{, 1}=\partial f / \partial e_{1}$ and $f_{, 5}=\partial f / \partial \gamma_{5}$. The nondimensional forms introduced in Eqs. (15)-(17) are defined by the following relationships:

$X_{1}=L \bar{X}_{1}, \quad t=\left(L / C_{s}\right) \bar{t}, \quad u_{j}=L \bar{u}_{j}, \quad$ and $v_{j}=C_{s} \bar{v}_{j}$.

The overbars denote nondimensional quantities, $L$ is defined to be the characteristic length of the shear pulse introduced into the solid, and $C_{s}$ is the linear shear wave speed of the material at zero prestrain; that is,

$$
C_{s}=\lim _{\gamma_{5} \rightarrow 0}\left[\sigma_{5,5}\left(\gamma_{5}\right) / \rho\right]^{1 / 2}
$$

In order to study the shear wave propagation in an incompressible material a slight reformulation is required. The determinant of the left Cauchy-Green tensor for incompressible materials is by definition equal to one, from which we conclude that the linear strain variable $e_{1}$ vanishes. Furthermore, the scalar quantity $\alpha_{0}$ in the constitutive relations (1) needs to be replaced by an arbitrary pressure $P$ which is determined by the motion of the material (Hunter, 1983). The constitutive relations therefore take the form

$$
\sigma_{i j}=-P \delta_{i j}+\alpha_{1} B_{i j}+\alpha_{2} B_{i r} B_{r j},
$$

where $\alpha_{1}$ and $\alpha_{2}$ are identical to those defined in Eq. (6). The shear mode can now be decoupled from the longitudinal mode. That is, the first equation in (14) imposes no constraint on the shear deformation but only provides the solu- tion for the arbitrary pressure $P$. This reduces the problem from a fourth-order system of equations to a second-order system. The resulting $2 \times 2$ speed matrix for an incompressible material reads

$$
\left[\begin{array}{cc}
0 & -\sigma_{5,5} / \rho_{0} C_{s}^{2} \\
-1 & 0
\end{array}\right]
$$

and the two-dimensional solution vector $w_{\eta}$ is

$$
\left[\begin{array}{l}
\bar{v}_{3} \\
\gamma_{5}
\end{array}\right] \text {. }
$$

The only component of the stress tensor required in (21) is $\sigma_{5}$ which may be found by setting $e_{1}=0$ in the last of (13), i.e.,

$$
\sigma_{5}=\gamma_{5}\left[\alpha_{1}+\alpha_{2}\left(2+\gamma_{5}^{2}\right)\right],
$$

which may be further reduced if the second and third of (6) are employed; the final result reads

$$
\sigma_{5}=\rho_{0} U^{\prime}
$$

where $U^{\prime} \equiv d U / d \gamma_{5}$. If (23) is substituted in (19) it is found that the simplified form of $C_{s}$ is

$$
C_{s}=\left[U^{\prime \prime}(0)\right]^{1 / 2} \text {. }
$$

\section{QUADRATIC NONLINEARITY}

To analyze the shear wave distortion caused by nonlinear effects it will be useful to employ perturbation methods to derive the evolution equations governing the propagation. The evolution equation derived here will be applicable to arbitrary initial wave profiles. In this analysis the system is subjected to small perturbations about some initial base state $w_{\alpha}^{(0)}$. The solution vector will be expanded as follows:

$$
w_{\alpha}=w_{\alpha}^{(0)}+\epsilon w_{\alpha}^{(1)}+\epsilon^{2} w_{\alpha}^{(2)}+O\left(\epsilon^{3}\right) .
$$

The quantity $\epsilon$ is a small parameter characterizing the disturbance amplitude. The undisturbed state is regarded to be at rest with an applied constant strain. The quantity $w_{\alpha}^{(0)}$ will therefore be given by $w_{1}^{(0)}=w_{2}^{(0)}=w_{3}^{(0)}=0$ and $w_{4}^{(0)}=\gamma_{5}^{(0)}$, where $\gamma_{s}^{(0)}$ is a constant. Here, we note that the shear wave pulse evolves linearly in the hyperelastic medium for times of order one and in a nonlinear manner for times of order 1/ $\epsilon$ and larger, see, e.g., Taniuti and Wei (1968). By substituting the expansion (25) into Eq. (15), by transforming from $\left(\bar{X}_{1}, \bar{t}\right)$ to wave coordinates $Y=\bar{X}_{1}-\lambda \bar{t}$ and $\tau^{\prime}=\bar{\epsilon} \bar{t}$, where $\lambda$ represents an eigenvector of the speed matrix, and by applying the technique of Taniuti and Wei (1968), we find that the strain is governed by the following evolution equation:

$$
\frac{\partial W}{\partial \tau^{\prime}}+\Gamma W \frac{\partial W}{\partial Y}=0,
$$

where $W\left(Y, \tau^{\prime}\right)$ is related to the lowest-order disturbance by

$$
w_{\alpha}^{(1)}=r_{\alpha} W
$$

and

$$
\begin{aligned}
& \Gamma \equiv l_{\alpha} B_{\alpha \beta v} r_{\beta} r_{v} / l_{\delta} r_{\delta} \\
& B_{\alpha \beta v} \equiv \frac{\partial A_{\alpha \beta}}{\partial w_{v}}\left(w_{\eta}^{(0)}\right) .
\end{aligned}
$$


The quantity $l_{\alpha}$ is the left eigenvector and $r_{\alpha}$ is the right eigenvector of the speed matrix evaluated at the base state; i.e., $A_{\alpha \beta}^{(0)} \equiv A_{\alpha \beta}\left(w_{\eta}^{(0)}\right)$. The scalar quantity $\Gamma$ is referred to as the first-order or quadratic steepening coefficient. Equation (26) is frequently referred to as the inviscid Burgers' equation.

The quantities that were defined in a general fashion by Eqs. (25)-(27) will now be expressed explicitly for the shear wave problem introduced in Sec. I. The eigenvalues $\lambda$ of the speed matrix $A_{\alpha \beta}^{(0)}$ are the nondimensional wave velocities of the shear and compressive modes. There exists two nondimensional wave speeds corresponding to each mode of propagation, that is, a positive and a negative component corresponding to a "right" or "left" moving wave. The specific eigenvalue of (17), which corresponds to a right moving shear wave, is found to be

$$
\begin{aligned}
\lambda_{s}= & \left(( 1 / 2 \rho _ { 0 } C _ { s } ^ { 2 } ) \left\{\sigma_{1,1}+\sigma_{5,5}-\left(\sigma_{1,1}-\sigma_{5,5}\right)\right.\right. \\
& \left.\left.\times\left[1+4 \sigma_{1,5} \sigma_{5,1} /\left(\sigma_{1,1}-\sigma_{5,5}\right)^{2}\right]^{1 / 2}\right\}\right)^{1 / 2}
\end{aligned}
$$

The term "shear" wave is appropriate in describing the above equation because it may easily be shown that $\lambda_{s}=\left(\sigma_{5,5} / \rho_{0}\right)^{1 / 2} / C_{s}=1$ at $\gamma_{5}^{(0)}=0$. The right eigenvector $r_{\alpha}$ corresponding to this eigenvalue $\lambda_{s}$ can be written as

$$
\left[\begin{array}{c}
-\lambda_{s} \sigma_{1,5}\left[\lambda_{s}^{2} \rho_{0} C_{s}^{2}-\sigma_{1,1}\right]^{-1} \\
-\lambda_{s} \\
\sigma_{1,5}\left[\lambda_{s}^{2} \rho_{0} C_{s}^{2}-\sigma_{1,1}\right]^{-1} \\
1
\end{array}\right] .
$$

In like manner, the left eigenvector $l_{\alpha}$ is found to be

$$
\begin{aligned}
& {\left[\sigma_{5,1}\left[\lambda_{s}^{2} \rho_{0} C_{s}^{2}-\sigma_{1,1}\right]^{-1} ; 1 ;\right.} \\
& \left.\quad-\lambda_{s} \sigma_{5,1}\left[\lambda_{s}^{2} \rho_{0} C_{s}^{2}-\sigma_{1,1}\right]^{-1} ;-\lambda_{s}\right] .
\end{aligned}
$$

The first-order steepening coefficient $\Gamma$ is found to be

$$
\begin{aligned}
\Gamma= & \left(-1 / r_{\alpha} l_{\alpha} \rho_{0} C_{s}^{2}\right)\left\{l _ { 1 } \left(\sigma_{1,11} r_{3}^{2}+2 \sigma_{1,15} r_{3} r_{4}\right.\right. \\
& \left.\left.+\sigma_{1,55} r_{4}^{2}\right)+l_{2}\left(\sigma_{5,11} r_{3}^{2}+2 \sigma_{5,15} r_{3} r_{4}+\sigma_{5,55} r_{4}^{2}\right)\right\},
\end{aligned}
$$

where $r_{\alpha} l_{\alpha}$ is, simply,

$$
r_{\alpha} l_{\alpha}=-2 \lambda_{s}\left[1+\sigma_{1,5} \sigma_{5,1} /\left(\lambda_{s}^{2} \rho_{0} C_{s}^{2}-\sigma_{1,1}\right)^{2}\right] .
$$

Expressions for the partial derivatives of the stress tensor (e.g., $\sigma_{1,11}, \sigma_{5,11}$, etc.) may be found in Carman and Cramer (1991). A form of these equations which is Eulerian based was presented by Nariboli and Lin (1973) when they investigated the shear wave propagation in an unstrained isotropic hyperelastic medium. They discovered that the first-order steepening coefficient $\Gamma$ presented in Eq. (31) is identically zero for all isotropic material systems if the undisturbed state is strain free. Through the use of the equations presented in Sec. I, it can be shown that the gradients $\partial \alpha_{0} / \partial \gamma_{5}$, $\partial \alpha_{1} / \partial \gamma_{5}$, and $\partial \alpha_{2} / \partial \gamma_{5}$ vanish at zero prestrain and thus reduce the quantities $\sigma_{1,5}$ and $\sigma_{5,1}$ to zero. If these values are introduced into Eqs. (29) and (30) along with the fact $\lambda_{s}\left(\gamma_{s}^{(0)}=0\right)=1$, it is found that $l_{\alpha}$ reduces to

$$
[0 ; 1 ; 0 ;-1]
$$

and $r_{\alpha}$ is found to be the negative of the transpose of $l_{\alpha}$. Evaluation of $\Gamma$ with these relationships reduces Eq. (31) to

$$
\lim _{\gamma_{s} \rightarrow 0} \Gamma=\sigma_{5,5 s} / 2 \rho_{0} C_{s}^{2}
$$

It may also readily be shown that $\sigma_{5,55}$ vanishes for $\gamma_{5}^{(0)}=0$ by similar arguments. Thus $\Gamma=0$, at $\gamma_{5}^{(0)}=0$, in complete agreement with Nariboli and Lin (1973) and Lee-Bapty (1981).

For an incompressible material, Eqs. (21)-(22) are used for the evaluation of $\lambda_{s}$ and $\Gamma$. This results in simplified forms of these expansions as compared to those presented in Eqs. (28) and (31). The reduced forms are found to be

$$
\begin{aligned}
& \lambda_{s}=\left(\frac{\sigma_{5,5}}{\rho_{0} C_{s}^{2}}\right)^{1 / 2}=\left(\frac{U^{\prime \prime}\left(\gamma_{s}^{(0)}\right)}{U^{\prime \prime}(0)}\right)^{1 / 2}, \\
& \Gamma=\frac{\sigma_{5,55}}{2 \rho_{0} C_{s}^{2} \lambda_{s}}=\frac{U^{\prime \prime \prime}\left(\gamma_{s}^{(0)}\right)}{2\left[U^{\prime \prime}\left(\gamma_{s}^{(0)}\right) U^{\prime \prime}(0)\right]^{1 / 2}} .
\end{aligned}
$$

A comparison of the dimensional wave speeds in the Lagrangian and Eulerian coordinate systems is provided in the Appendix. One should note that the wave speeds are equal in either of the coordinate systems employed, at least within the accuracy of the present theory.

\section{CUBIC NONLINEARITY}

As pointed out in the Introduction, we will refer to base states $\gamma_{5}^{(0)}$ which correspond to values of $\Gamma=0$ as critical states or points. The shear pulse at these base states evolves linearly even for times $O(1 / \epsilon)$ and in a nonlinear manner for times $O\left(1 / \epsilon^{2}\right)$ and larger. The details of the evolution at these higher times requires inclusion of terms which were properly neglected in the $\Gamma=0(1)$ approximation of Taniuti and Wei (1968). It turns out that a cubic form of Eq. (26) is required to describe the wave dynamics in the vicinity of a critical point. Cramer and Sen (1991) have shown that the appropriate extension of Eq. (26) is given by

$$
\frac{\partial W}{\partial \tau}+\left(\hat{\Gamma}+\frac{1}{2} \Lambda W\right) W \frac{\partial W}{\partial Y}=0,
$$

where $\tau=\epsilon^{2} \bar{t}$ and

$$
\begin{aligned}
\hat{\Gamma} & \equiv \Gamma / \epsilon, \\
\Lambda & \equiv\left(\frac{2 l_{\alpha} C_{\alpha \beta v \gamma} r_{\beta} r_{v} r_{\gamma}-\left(2 d_{A}+f_{A}\right) B_{A \beta v} r_{\beta} r_{v}}{l_{\delta} r_{\delta}}\right) .
\end{aligned}
$$

In the above equations, $C_{\alpha \beta v \gamma}$ is determined by the higherorder derivatives of the speed matrix as follows:

$$
C_{\alpha \beta v \gamma}=\frac{1}{2} \frac{\partial^{2} A_{\alpha \beta}}{\partial w_{v} \partial w_{\gamma}}\left(w_{\delta}^{(0)}\right) .
$$

The vectors $d_{A}$ and $f_{A}$ in Eq. (37) are found by solving

$$
R_{A J} d_{J}=l_{\eta} B_{\eta \alpha \gamma} r_{\gamma} a_{A \alpha}, \quad R_{A J} f_{J}=l_{\eta} B_{\eta \gamma \alpha} r_{\gamma} a_{A \alpha},
$$

where

$$
R_{A J}=a_{A \alpha} a_{J \alpha}
$$

and $a_{A \alpha}$ represents a nonsquare matrix whose rows are comprised of the three independent row vectors of $\left(A_{\alpha \beta}^{(0)}-\lambda \delta_{\alpha \beta}\right)$. For all subsequent formula description, only the row space corresponding to the eigenvalue (28) is addressed. The independent rows of $\left(A_{\alpha \beta}^{(0)}-\lambda \delta_{\alpha \beta}\right)$ will be taken to be $a_{1 \alpha}, a_{3 \alpha}$, and $a_{4 \alpha}$. That is, the subscripted upper- 
case latin indices will range over 1,3, and 4. In Eq. (36), $\Lambda$ is normally referred to as the second-order or cubic nonlinearity parameter. For a compressible material represented by Eqs. (16) and (17), this parameter is found to be

$$
\begin{aligned}
\Lambda= & \left(1 / l_{\alpha} r_{\alpha}\right)\left\{( - 1 / \rho _ { 0 } C _ { s } ^ { 2 } ) \left[l _ { 1 } \left(\sigma_{1,111} r_{3}^{3}+3 \sigma_{1,115} r_{3}^{2} r_{4}\right.\right.\right. \\
& \left.+3 \sigma_{1,551} r_{4}^{2} r_{3}+\sigma_{1,555} r_{4}^{3}\right)+l_{2}\left(\sigma_{5,111} r_{3}^{3}\right. \\
& \left.\left.+3 \sigma_{5,115} r_{3}^{2} r_{4}+3 \sigma_{5,551} r_{4}^{2} r_{3}+\sigma_{5,555} r_{4}^{3}\right)\right] \\
& \left.+\left(3 d_{1} / \rho_{0} C_{s}^{2}\right)\left[\sigma_{1,11} r_{3}^{2}+2 \sigma_{1,15} r_{3} r_{4}+\sigma_{1,55} r_{4}^{2}\right]\right\} .
\end{aligned}
$$

Explicit expressions for the higher-order derivatives of the stress tensor (e.g., $\sigma_{1,111}, \sigma_{5,555}$, etc.) may be found in Carman and Cramer (1991). The quantity $d_{1}$ in the above equation may be found by solving (39). The result is as follows:

$d_{1}=\left(g_{1} R_{33}-R_{13} g_{3}-R_{14} g_{4}\right) /\left(R_{11} R_{33}-R_{13}^{2}-R_{14}^{2}\right)$,

where the matrix $R_{A J}$ can be shown to be

$$
\left[\begin{array}{ccc}
\lambda_{s}^{2}+\frac{1}{\rho_{0}^{2} C_{s}^{4}}\left(\sigma_{1,1}^{2}+\sigma_{1,5}^{2}\right) & \frac{\lambda_{s} \sigma_{1,1}}{\rho_{0} C_{s}^{2}}+\lambda_{s} & \frac{\lambda_{s} \sigma_{1,5}}{\rho_{0} C_{s}^{2}} \\
\frac{\lambda_{s} \sigma_{1,1}}{\rho_{0} C_{s}^{2}}+\lambda_{s} & 1+\lambda_{s}^{2} & 0 \\
\frac{\lambda_{s} \sigma_{1,5}}{\rho_{0} C_{s}^{2}} & 0 & 1+\lambda_{s}^{2}
\end{array}\right]
$$

and the vector $g_{A}$ is defined to be

$$
\begin{aligned}
g_{1}= & \left(\sigma_{1,1} / \rho_{0}^{2} C_{s}^{4}\right)\left[l_{1}\left(\sigma_{1,11} r_{3}+\sigma_{1,15} r_{4}\right)\right. \\
& \left.+l_{2}\left(\sigma_{5,11} r_{3}+\sigma_{5,15} r_{4}\right)\right] \\
& +\left(\sigma_{1,5} / \rho_{0}^{2} C_{s}^{4}\right)\left[l_{1}\left(\sigma_{1,51} r_{3}+\sigma_{1,55} r_{4}\right)\right. \\
& \left.+l_{2}\left(\sigma_{5,51} r_{3}+\sigma_{5,55} r_{4}\right)\right], \\
g_{3}= & \left(\lambda_{s} / \rho_{0} C_{s}^{2}\right)\left[l_{1}\left(\sigma_{1,11} r_{3}+\sigma_{1,15} r_{4}\right)\right] \\
& \left.+l_{2}\left(\sigma_{5,11} r_{3}+\sigma_{5,15} r_{4}\right)\right], \\
g_{4}= & \left(\lambda_{s} / \rho_{0} C_{s}^{2}\right)\left[l_{1}\left(\sigma_{1,51} r_{3}+\sigma_{1,55} r_{4}\right)\right. \\
& \left.+l_{2}\left(\sigma_{5,51} r_{3}+\sigma_{5,55} r_{4}\right)\right] .
\end{aligned}
$$

At the critical point corresponding to zero prestrain $\gamma_{5}^{(0)}=0$, it can be shown that Eq. (40) reduces to the following:

$$
\Lambda=\frac{1}{2}\left(\frac{1}{\rho_{0} C_{s}^{2}} \sigma_{5,555}+\frac{3 \sigma_{5,15} \sigma_{1,55}}{\left(\rho_{0}^{2} C_{s}^{4}-\sigma_{1,1} \rho_{0} C_{s}^{2}\right)}\right),
$$

where each quantity should be evaluated at the $\gamma_{5}^{(0)}=0$ state.

For an incompressible material, Eqs. (21) and (22) are used for the calculation of $\Lambda$ in Eq. (37). This parameter is expressed in a much simpler form than that presented in Eq. (40). For arbitrary $\gamma_{s}^{(0)}$, the following equation can easily be derived:

$\Lambda=\frac{\sigma_{5,55 s}}{2 \rho_{0} C_{s}^{2} \lambda_{s}}=\frac{U^{(i v)}\left(\gamma_{s}^{(0)}\right)}{2\left(U^{\prime \prime}\left(\gamma_{s}^{(0)}\right) U^{\prime \prime}(0)\right)^{1 / 2}}$.

To summarize, the evolution of the lowest-order distur- bance will be governed by (26) when $\Gamma=0(1)$. We anticipate this to be the case for most configurations involving nonzero prestrain. When $\Gamma$ is of order $\epsilon$, the wave distortion will only be noticeable over time scales of order $\epsilon^{-2}$ and will therefore be governed by (36). Specifically, this occurs in the vicinity of the critical point corresponding to $\gamma_{s}^{(0)}=0$. It is also possible that other critical points exist for nonzero. prestrains. Whether this later possibility does indeed occur can only be determined by a consideration of free energy functions specific to the material of interest. The material model will also provide information regarding the sign of $\Gamma$, and, therefore, the qualitative behavior in prestrained configurations can be determined. In Secs. V and VI specific material models will be employed to generate numerical values for $\Gamma$ and $\Lambda$.

\section{SHOCK FORMATION AND DISSIPATION}

The local wave speed corresponding to (26) may be found by a straightforward application of the method of characteristics and can be written as

$$
C_{s}\left[\lambda_{s}+\Gamma\left(\gamma_{5}-\gamma_{s}^{(0)}\right)\right] \text {, }
$$

where $C_{s}$ is given by (19), $\lambda_{s}$ is given by (28) or the first of (35) and $\Gamma$ is given by (31) or (35). In like manner, the dimensional wave speed corresponding to (36) is found to be

$$
\boldsymbol{C}_{s}\left[\lambda_{s}+\Gamma\left(\gamma_{5}-\gamma_{s}^{(0)}\right)+(\Lambda / 2)\left(\gamma_{5}-\gamma_{5}^{(0)}\right)^{2}\right],
$$

where $\Lambda$ is given by (40) or (45). Because $\gamma_{5}$ varies from point to point in the wave, the propagation speed also varies from point to point, thus resulting in a distortion and steepening of most smooth pulses. To provide an example of this distortion, we have plotted exact solutions to (26) for a value of $\Gamma=-0.5$ in Fig. 1. This numerical value corresponds to the compressible foamed polyurethane described in Sec. VI at a prestrain of $\gamma_{s}^{(0)}=0.2$. At the scaled times $\tau^{\prime}=0.5$ and 1.0, the solutions are seen to be triple valued. Such solutions are physically unrealistic. In real materials, the large strain gradients that occur just before the overturning takes place will trigger physical mechanisms capable of counteracting the nonlinear distortion. These may include both dis-

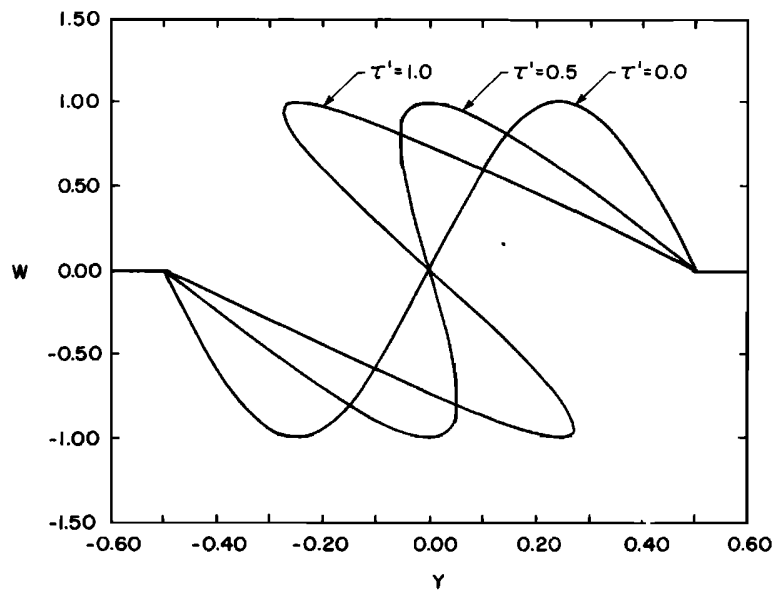

FIG. 1. The wave evolution of a sine pulse in compressible foamed polyurethane rubber at $\gamma_{5}^{(0)}=0.2(\Gamma \approx-0.5)$. 
sipation and dispersion. Here, we follow Nariboli and Lin (1973) and Lee-Bapty (1981) in restricting our attention to dissipation of the Kelvin-Voigt type. In following these previous investigations we simply add linear viscous terms to the elastic stress tensor (1). The modified stress tensor then reads

$$
\hat{\sigma}_{i j}=\sigma_{i j}+\mu^{\prime}{ }_{v} \frac{\partial v_{l}}{\partial X_{l}} \delta_{i j}+\mu_{v}\left(\frac{\partial v_{i}}{\partial X_{j}}+\frac{\partial v_{j}}{\partial X_{i}}\right),
$$

where the constants $\mu_{v}$ and $\mu_{v}^{\prime}$ are viscosities and $\sigma_{i j}$ is the elastic tensor given by (1). Because only the lowest-order term for the dissipation is required, expressions involving the derivatives with respect to $x_{i}$ in the viscous stresses have been replaced by those with respect to $X_{i}$. The modifications to the lossless equations of Secs. II and III may be derived by the techniques of Taniuti and Wei (1968) and Cramer and Sen (1991), respectively. The dissipative versions of Eqs. (26) and (36) are found to be

$$
\frac{\partial W}{\partial \tau^{\prime}}+\Gamma W \frac{\partial W}{\partial Y}=\frac{\delta}{2} \frac{\partial^{2} W}{\partial Y^{2}}
$$

and

$$
\frac{\partial W}{\partial \tau}+\left(\hat{\Gamma}+\frac{\Lambda}{2} W\right) W \frac{\partial W}{\partial Y}=\frac{\hat{\delta}}{2} \frac{\partial^{2} W}{\partial Y^{2}},
$$

where

$$
\begin{aligned}
\delta \equiv & \frac{\mu_{v}}{\epsilon \rho_{0} C_{s} L} \\
& \times \frac{1+\left\{\sigma_{1,5} \sigma_{5,1} /\left[\left(\lambda_{s}\right)^{2} C_{s}^{2} \rho_{0}-\sigma_{1,1}\right]^{2}\right\}\left(2+\mu_{v}^{\prime} / \mu_{v}\right)}{1+\left\{\sigma_{1,5} \sigma_{5,1} /\left[\left(\lambda_{s}\right)^{2} C_{s}^{2} \rho_{0}-\sigma_{1,1}\right]^{2}\right\}}
\end{aligned}
$$

and $\hat{\delta} \equiv \delta / \epsilon$. Here, each term in $\delta$ is to be evaluated at the undisturbed state. Equation (49) is recognized as the usual Burgers' equation and (50) is the cubic version of the equation derived by Cramer and Kluwick (1984) in the context of ordinary gasdynamics, Cramer and Sen (1990) in the context of superfluid hydrodynamics as well as the general analysis of Cramer and Sen (1991). The $\Gamma=0$ version of (50) has been derived by Nariboli and Lin (1973) and LeeBapty (1981) in a context similar to that here. Morris and Nariboli (1972) also found that $\Gamma=0$ version governs pulse propagation in applications involving elastic dielectrics.

We also note that the dissipation parameter (51) reduces to that obtained by the previous investigators in the $\gamma_{5}^{(0)}=0$ limit. The term involving the longitudinal viscosity $\mu_{v}^{\prime}+2 \mu_{v}$ is to be expected when it is recalled that the longitudinal strain perturbation $e_{1}$ is nonzero when the prestrain $\gamma_{s}^{(0)}$ is nonzero. Inspection of (51) also reveals that the dissipation parameter is modified by the prestrain. This will influence the shock thickness whenever the strain is of order one, including those cases near a second critical point.

In order that the dissipation in (49) and (50) be of the same order as the nonlinear distortion, the wave Reynolds number $\rho_{0} C_{s} L / \mu_{v}$ must be either of order $\epsilon^{-1}$ for the classical $\Gamma=0(1)$ theory or of order $\epsilon^{-2}$ for the $\Gamma=O(\epsilon)$ theory. This requires the length scale $L$ be of order

$$
\frac{1}{\epsilon} \frac{\mu_{v}}{\rho_{0} C_{s}} \text { or } \frac{1}{\epsilon^{2}} \frac{\mu_{v}}{\rho_{0} C_{s}}
$$

If $L$ is the typical length scale of the signal and if it satisfies (52) then $\delta=0(1)$ or $\hat{\delta}=O(1)$ and dissipative effects will be noticable over most of the pulse. If, on the other hand, the pulse is considerably longer than the length indicated in (52), then $\delta \ll 1$ in the $\Gamma=O(1)$ theory or $\hat{\delta} \ll 1$ in the $\Gamma=O(\epsilon)$ theory. Dissipative effects will then be negligible over most of the pulse and either (26) or (36) will govern the initial stages of the distortion. However, dissipative effects will nevertheless be important in regions of large gradients generated by the steepening. These narrow regions are recognized as shock waves and will have thicknesses on the order of the lengths indicated in (52). If the shocks are thin enough, they can be modeled as discontinuities. The appropriate dimensional shock speed corresponding to $(49)$ is known to be

$$
C_{s}\left[\lambda_{s}+\Gamma\left(\gamma_{5}^{+}+\gamma_{5}^{-}\right) / 2\right],
$$

where the superscripts \pm refer to the properties just after and just before the discontinuity. The dimensional shock speed corresponding to (50) can be shown to be

$$
\begin{gathered}
C_{s}\left\{\lambda_{s}+\Gamma\left[\left(\gamma_{s}^{+}+\gamma_{s}^{-}\right) / 2\right]+(\Lambda / 6)\left[\left(\gamma_{s}^{+}-\gamma_{s}^{(0)}\right)^{2}\right.\right. \\
\left.\left.+\left(\gamma_{s}^{+}-\gamma_{s}^{(0)}\right)\left(\gamma_{s}^{-}-\gamma_{s}^{(0)}\right)+\left(\gamma_{s}^{-}-\gamma_{s}^{(0)}\right)^{2}\right]\right\} .
\end{gathered}
$$

An approximate graphical representation of the shock using Landau's equal area rule can be employed to model the shock profile. The construction of a pulse profile using this methodology is depicted in Fig. 2.

In closing, we note that the dissipation parameters $\hat{\delta}$ and $\delta$ must be modified when the material is incompressible. Under these conditions, the parameter corresponding to $\delta=\epsilon \hat{\delta}$ is

$$
\mu_{v} / \epsilon \rho_{0} C_{s} L \text {. }
$$

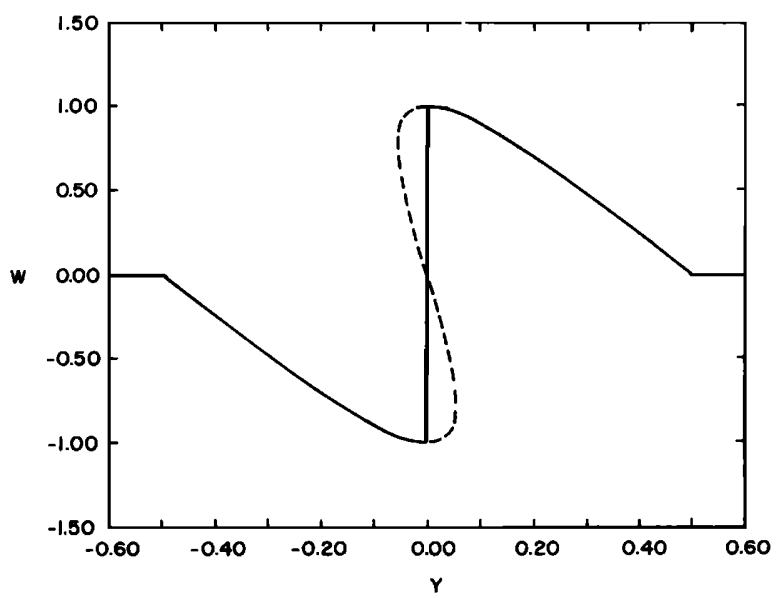

FIG. 2. Wave profile obtained by using Landau's equal area rule. Dotted line denotes overturned profile identical to that of Fig. 1 at $\tau^{\prime}=0.5$. Solid line denotes actual profile after shock fitting. 


\section{EXAMPLE-OGDEN'S MODEL FOR INCOMPRESSIBLE MATERIALS}

A typical example of an incompressible hyperelastic isotropic material is natural vulcanized rubber. Constitutive modeling of rubber has received a great deal of attention in the literature due to its wide use in both industrial and scientific applications. The well-known constitutive model of Mooney-Rivlin (see Treloar, 1975) has been utilized by a number of authors in studying the behavior of various incompressible rubber systems. However, this model, while providing accurate correlation with experimental results below stretches of the order two, significantly deviates from test data at larger stretches in uniaxial loadings; this deviation is depicted in Fig. 3. To remedy this, Ogden (1972) proposed a higher-order constitutive model that represents the free energy as an expansion of the principal stretches. In comparing the accuracy of the Ogden model to the experimental results of Treloar, a high degree of correlation is found to exist between the two, up to and including stretches on the order of 7. A comparison between Ogden's theory and Treloar's experimental results has been reproduced in Fig. 3 . Additionally, the Ogden model not only provides highly accurate constitutive relations for uniaxial tension but is also accurate for biaxial tension and simple shear. It should also be noted that the Ogden model reduces to the much simpler Mooney-Rivlin model by appropriate choices of material parameters. Because of its accuracy and relative simplicity, the Ogden model will be employed in the following study to compute the nonlinearity parameters for natural rubber.

Ogden proposed that the free energy of an incompressible material can be represented as a series sum of the principal stretches. In particular, for vulcanized natural rubber one finds the following expression:

$$
U=\frac{1}{\rho} \sum_{n=1}^{3} \frac{\mu_{n}\left(a_{1}^{\chi_{n}}+a_{2}^{\chi_{n}}+a_{3}^{\chi_{n}}-3\right)}{\chi_{n}},
$$

where $a_{1}, a_{2}$, and $a_{3}$ represent the principal stretches and $\mu_{n}$ and $\chi_{n}$ represent material constants. The specific material parameters $\mu_{n}$ and $\chi_{n}$ in the Ogden model corresponding to vulcanized natural rubber were given as (Ogden, 1972)

$\chi_{1}=1.3, \quad \chi_{2}=5.0, \quad \chi_{3}=-2.0$,

$\mu_{1}=0.61762, \quad \mu_{2}=0.001176, \quad \mu_{3}=-0.09803 \mathrm{MPa}$.

For the deformations considered here, the principal stretches are given by

$$
a_{1}=1 / a_{2}=\left[\gamma_{5}+\left(\gamma_{5}^{2}+4\right)^{1 / 2}\right] / 2, \quad a_{3}=1 .
$$

The shear stress $\sigma_{5}$ for a simple shear problem can therefore be written as follows:

$$
\sigma_{5}=\sum_{n=1}^{3} \mu_{n} \frac{\left[0.5\left(\gamma_{5}+\left(4+\gamma_{5}^{2}\right)^{1 / 2}\right]^{\chi_{n}}-\left[0.5\left(\gamma_{5}+\left(4+\gamma_{5}^{2}\right)^{1 / 2}\right]^{-\chi_{n}}\right.\right.}{\left[0.5\left(\gamma_{5}+\left(4+\gamma_{5}^{2}\right)^{1 / 2}\right)\right]+\left[0.5\left(\gamma_{5}+\left(4+\gamma_{5}^{2}\right)^{1 / 2}\right)\right]^{-1}}
$$

The parameters $\Gamma$ and $\Lambda$ may be computed directly by substituting the appropriate derivatives of (57) into Eqs. (35) and (45). A plot of these values versus prestrain $\gamma_{5}^{(0)}$ is presented in Fig. 4 for the vulcanized natural rubber. At zero prestrain $\Gamma=0$ and the second-order nonlinearity coefficient is nonzero and negative $(\Lambda \approx-0.269)$. For nonzero but small prestrain $\Gamma<0$ which results in backward steepening waves. However, at larger prestrains $\Gamma>0$ and the wave steepens forward. Thus a second critical point $\Gamma=0$ is found at a prestrain of approximately 2.27 for this shear wave problem. Note that, at this prestrain, the second-order steepening coefficient $\Lambda=0.183$ has a sign which is the opposite of that at zero prestrain. In the neighborhood of these critical values, the cubic equation (36) governs the dynamics of the wave evolution and one would expect different wave evolution characteristics (i.e., dual shock formation) in these neighborhoods.

From the preceeding results, one can readily surmise that for incompressible materials the qualitative behavior of the shear wave evolution can be inferred from a diagram depicting the variation of the Cauchy stress versus linear shear strain. If the material softens, then $\sigma_{5,55}<0$. The value of $\Gamma$ will then be negative and the wave will steepen backward. If the material hardens $\sigma_{5,55}>0$, in which case $\Gamma$ is positive and the wave steepens forward. Near inflection points of the stress-strain curve $\Gamma$ is small and the cubic equation is required to describe the wave evolution. There- fore, plots similar to Fig. 3 could be used to deduce the qualitative behavior when the material is incompressible.

In Figs. 5-13, we present the wave evolutions for increasing values of prestrain. The initial wave profile was tak-
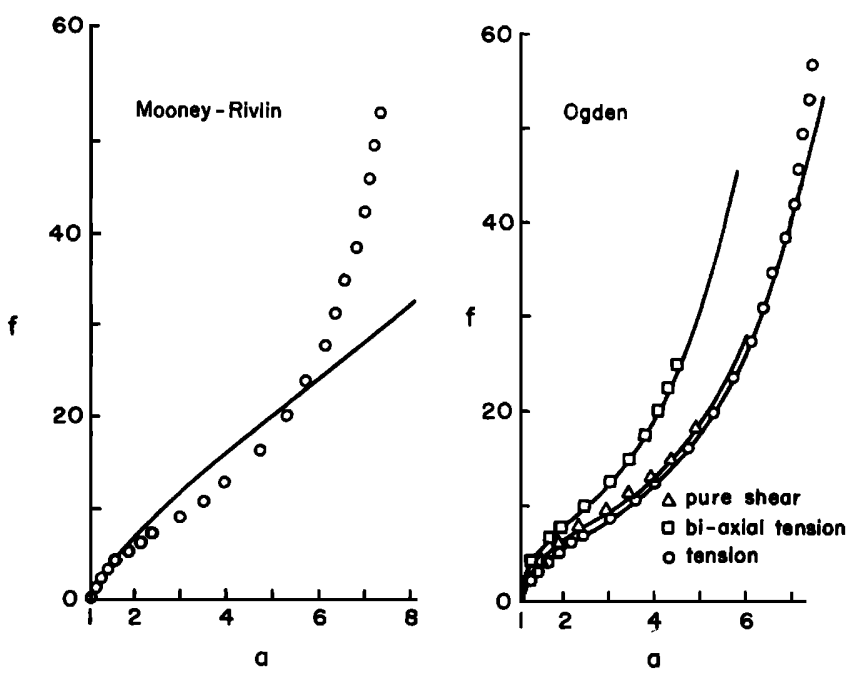

FIG. 3. Comparison between the theoretical models of Mooney-Rivlin and Ogden to the experimental results of Treloar. In figure at left the tensile test results (circles) of Treloar are compared to the theoretical predictions of Mooney-Rivlin. At right, Ogden's theory (solid lines) is compared to Treloar's results for three different loading modes. 


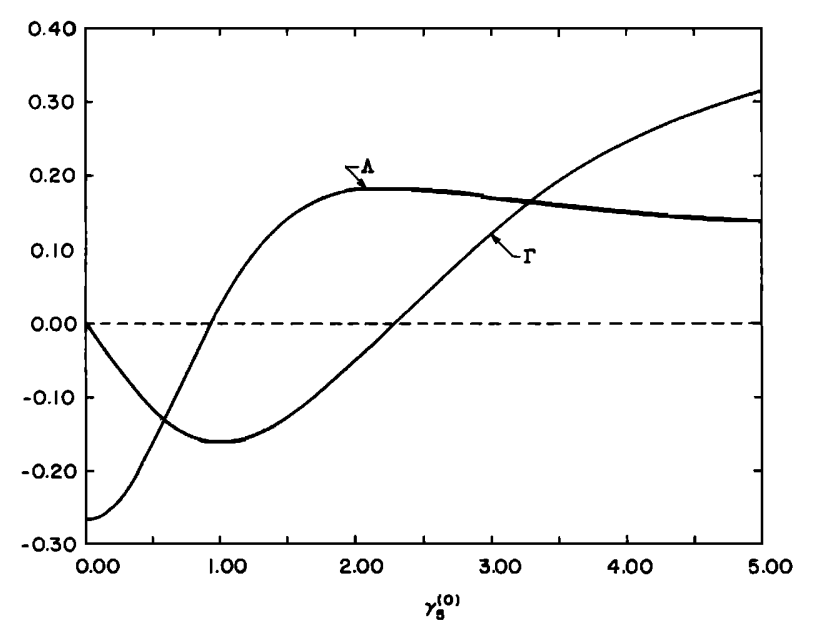

FIG. 4. $\Gamma$ and $\Lambda$ as a function of shear prestrain for vulcanized natural rubber represented by the Ogden model.

en to be sinusoidal with a wave amplitude parameter $\epsilon=0.1$ in all plots.

Figure 5 depicts the evolution of a propagating shear pulse at zero prestrain. This corresponds to the case considered by Nariboli and Lin (1973) and Lee-Bapty (1981). For this particular case, two shocks are formed. The first shock develops near the middle and is qualitatively similar to a backward steepening wave. The second shock develops at the wave's tail and in this region of the wave the behavior is similar to that seen in a forward steepening wave. This distorted profile has been previously referred to as a shark-fin configuration by Garrett (1981).

In Figs. 6-8, wave evolutions for prestrains in the vicinity of the unstrained case are illustrated; i.e., $\gamma_{5}^{(0)}=0.03,0.06$, and 0.09. With regard to Fig. 6, one notes the presence of a stationary node formed at a value of $W \simeq-0.60$. This point corresponds to a portion of the wave that is traveling at the same speed as the linearized wave speed $\lambda_{s}$. That is, the local wave speed $W[\hat{\Gamma}+\Lambda(W / 2)]$ vanishes. The case $\gamma_{s}^{(0)}=0.06$ is represented in Fig. 7 , and a node is seen at

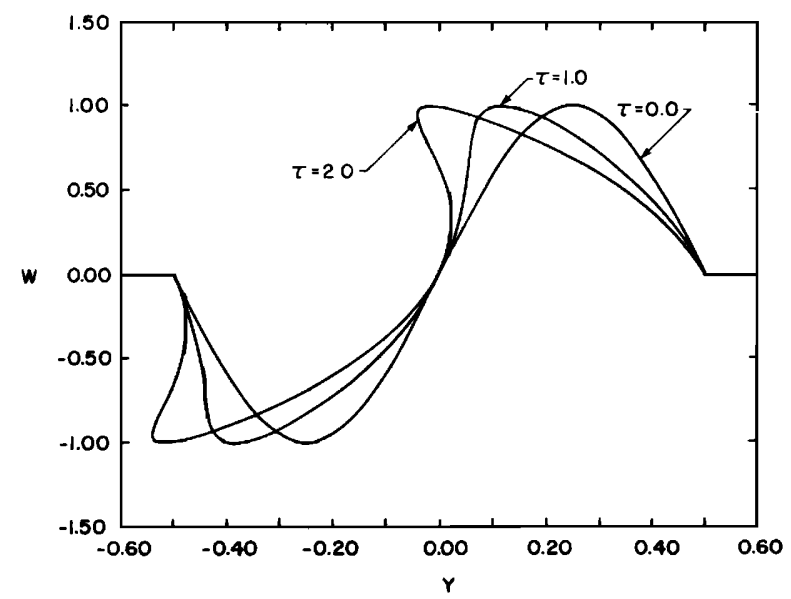

FIG. 5. The wave evolution of a shear sine pulse in vulcanized natural rubber at $\gamma_{5}^{(0)}=0.0$ prestrain $(\Lambda=-0.2685$ and $\Gamma=0.0)$.

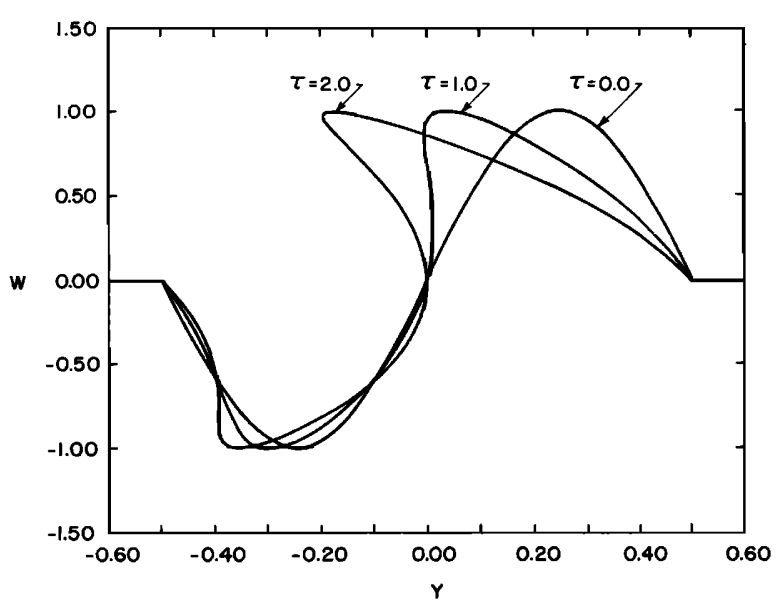

FIG. 6. The wave evolution of a shear sine pulse in vulcanized natural rubber at $\gamma_{5}^{(0)}=0.03$ prestrain $\left(\Lambda=-0.2685\right.$ and $\left.\Gamma=-8.05 \times 10^{-3}\right)$.

$W \simeq-1.0$. As for Fig. 8, no $W \neq 0$ stationary points are present as were in Figs. 6 and 7. Another point of significant interest concerns the positions at which the local steepening coefficient, i.e., $\hat{\Gamma}+\Lambda W$, vanishes. This represents a demarcation between the regions exhibiting local forward and backward steepening behavior. In Fig. 6 , this point occurs at $W \approx-0.30$, in Fig. 7 , it occurs at $W \approx-0.60$, and in Fig. 8, it occurs at $W \approx-0.90$. The changes in the position of the $\widehat{\Gamma}+\Lambda W=0$ point illustrates the trend toward pure backward steepening as the prestrain is increased.

The above figures have illustrated the evolution that occurs in the neighborhood of the $\gamma_{5}^{(0)}=0$ critical point. For prestrain values far removed from the critical points, the strictly quadratic equation (26) governs the wave distortion. We have computed and plotted the distortion using (26) for the case $\gamma_{5}^{(0)}=1.15$ in Fig. 9. For this case, $\Gamma \approx-0.158$ and the wave steepens backward. The single shock develops from steepening of both the top and bottom of the pulse at similar rates. This results in a centrally located shock.

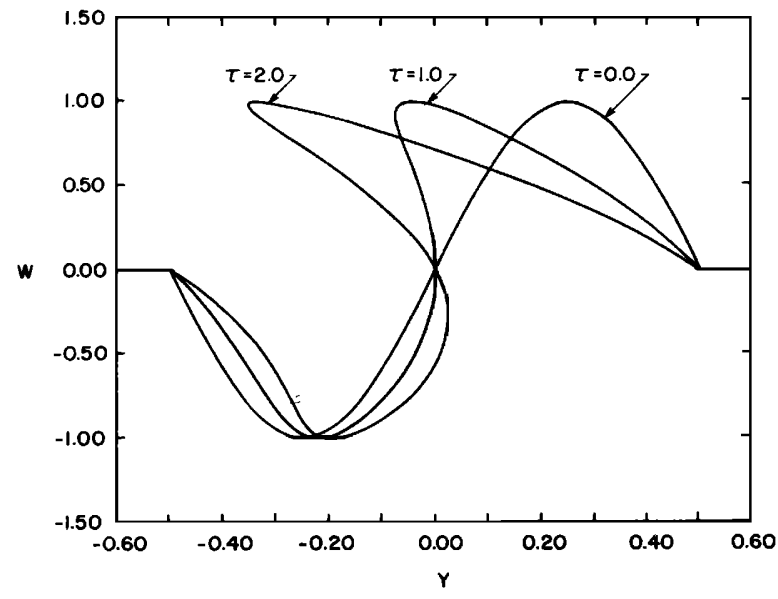

FIG. 7. The wave evolution of a shear sine pulse in vulcanized natural rubber at $\gamma_{s}^{(0)}=0.06$ prestrain $\left(\Lambda=-0.2685\right.$ and $\left.\Gamma=-1.608 \times 10^{-2}\right)$. 


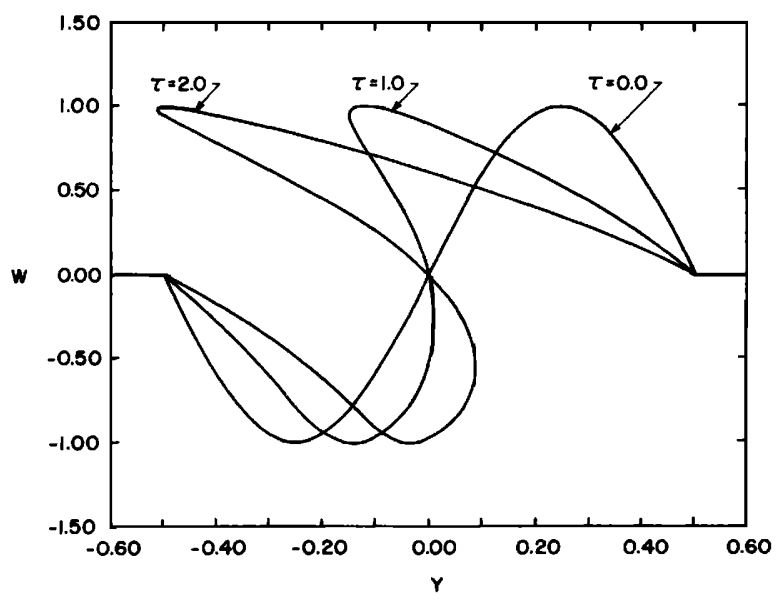

FIG. 8. The wave evolution of a shear sine pulse in vulcanized natural rubber at $\gamma_{5}^{(0)}=0.09$ prestrain $\left(\Lambda=-0.2685\right.$ and $\left.\Gamma=-2.406 \times 10^{-2}\right)$.

In Figs. 10-12, we illustrate the wave formation which occurs at strain values in the neighborhood of the second critical point $\gamma_{5}^{(0)}=2.27$. For a prestrain less than the critical value the undisturbed state still corresponds to $\Gamma<0$; a typical case is depicted in Fig. 10. Here, a part of the $W>0$ portion of the wave extends into the $\Gamma>0$ region. It is also seen that the local wave speed becomes greater than the linear wave speed for $W>0.6$. For wave propagation at the critical prestrain point, the evolution is depicted in Fig. 11. In Fig. 12, the wave evolution for a prestrain larger than the critical value is presented and a node is now seen at $W \cong-0.6$. In Figs. $10-12$, the local steepening coefficient vanishes at $W \approx 0.25, W=0$, and $W \approx-0.36$, respectively. This trend suggests, and is clearly depicted in the three figures, that the wave evolution becomes similar to a purely forward steepening wave for increasing prestrain values in the vicinity of the second critical state. In comparing the wave evolution presented for the second critical point, i.e., Figs. 10-12, as compared to the first critical point, i.e., Fig.

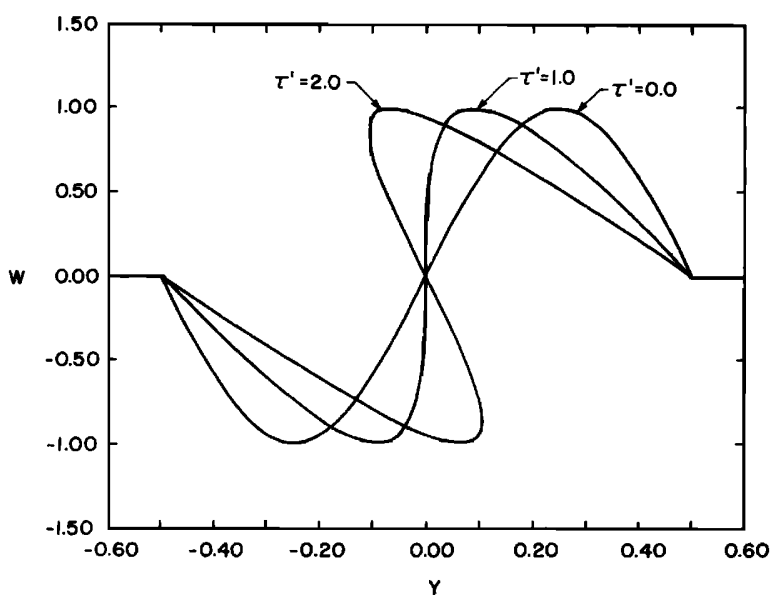

FIG. 9. The wave evolution of a shear sine pulse in vulcanized natural rubber at $\gamma_{s}^{(0)}=1.15$ prestrain $(\Gamma=-0.15817)$.

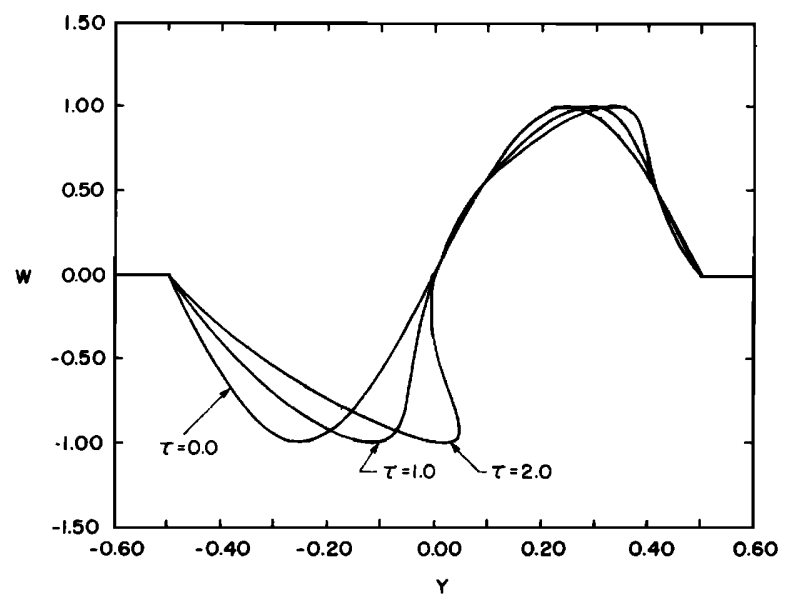

FIG. 10. The wave evolution of a shear sine pulse in vulcanized natural rubber at $\gamma_{5}^{(0)}=2.25$ prestrain $\left(\Lambda=0.182\right.$ and $\left.\Gamma=-4.59 \times 10^{-3}\right)$.

6-8, one notes a distinct difference in the shock formation. In Figs. 10-12, the dual shock formations are similar to a forward steepening shock for the top portion, i.e., $W>0$, and backward steepening shocks for the lower portion, i.e., $W<0$, of the wave. This behavior is qualitatively different than those presented for the wave evolution in the vicinity of zero prestrain. For even larger prestrains the steepening is strictly forward. A typical case, which employs (26), is illustrated in Fig. 13.

In summary, use of this specific model demonstrates the wide range of behavior to be expected as the prestrain is increased. Although the mixed convexity of the standard stress-strain diagram of rubber gives some hint of the complexity found, the present study shows definitively that both forward and backward steepening is possible. Furthermore, it has been shown that the nonlinear behavior in the neighborhood of the second critical point is qualitatively different than that at the critical point associated with the unstrained state.

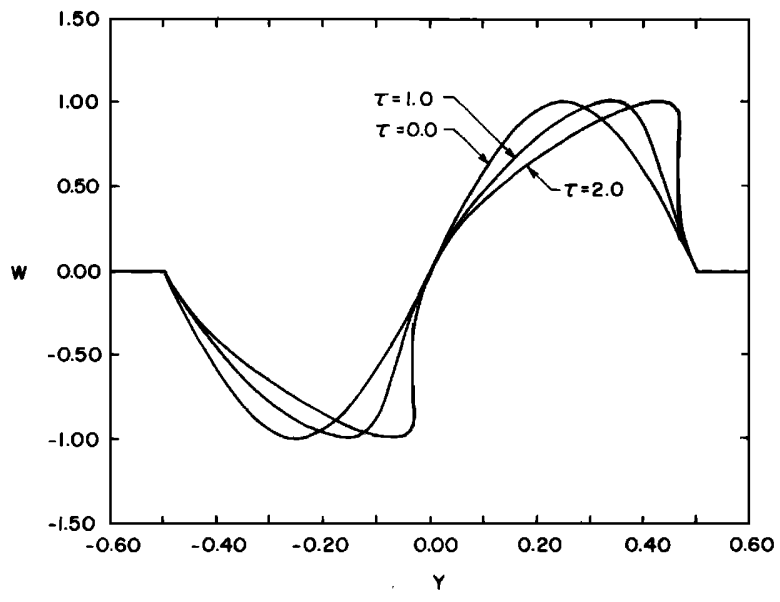

FIG. 11. The wave evolution of a shear sine pulse in vulcanized natural rubber at $\gamma_{5}^{(0)}=2.27$ prestrain $(\Lambda=0.182$ and $\Gamma=0.0)$. 


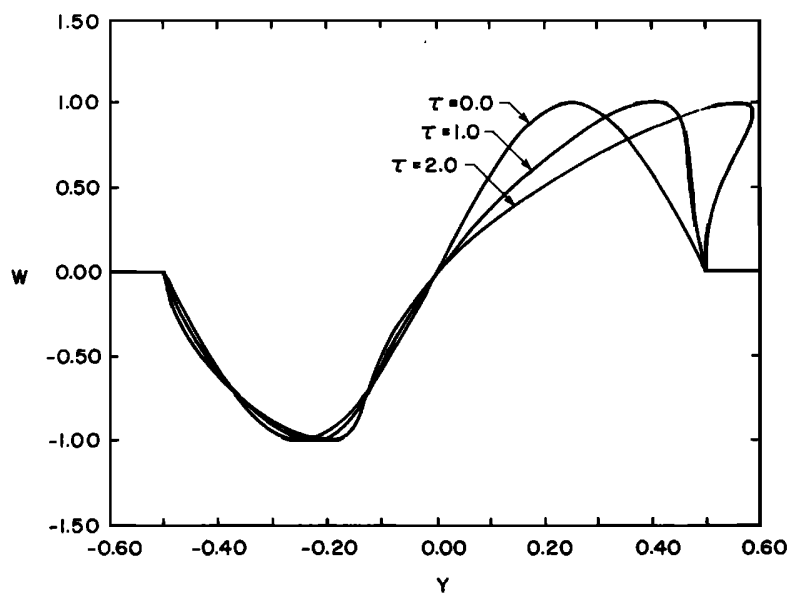

FIG. 12. The wave evolution of a shear sine pulse in vulcanized natural rubber at $\gamma_{s}^{(0)}=2.31$ prestrain $\left(\Lambda=0.182\right.$ and $\left.\Gamma=6.48 \times 10^{-3}\right)$.

\section{EXAMPLE-BLATZ AND KO'S MODEL FOR COMPRESSIBLE MATERIALS}

A typical example of a common compressible material which is capable of large nonlinear deformations is foamed polyurethane rubber. This isotropic material was extensively studied by Blatz and Ko (1962) who postulated the existence of a specific form of the free energy function which includes a dependence on the determinant of the left Cauchy-Green tensor $\mathrm{III}_{B}$, thus taking into account volume changes. The free energy function was taken to be

$$
\begin{aligned}
U= & \frac{\mu \beta}{2 \rho_{0}}\left(\mathrm{I}_{B}+\frac{1-2 v}{v}\left(\mathrm{III}_{B}^{-v /(1-2 v)}-1\right)-3\right) \\
& +\frac{\mu(1-\beta)}{2 \rho_{0}} \\
& \times\left(\frac{\mathrm{II}_{B}}{\mathrm{III}_{B}}+\frac{1-2 v}{v}\left(\mathrm{III}_{B}^{v /(1-2 v)}-1\right)-3\right),
\end{aligned}
$$

where $\mu, v$, and $\beta$ are material constants. It is interesting to

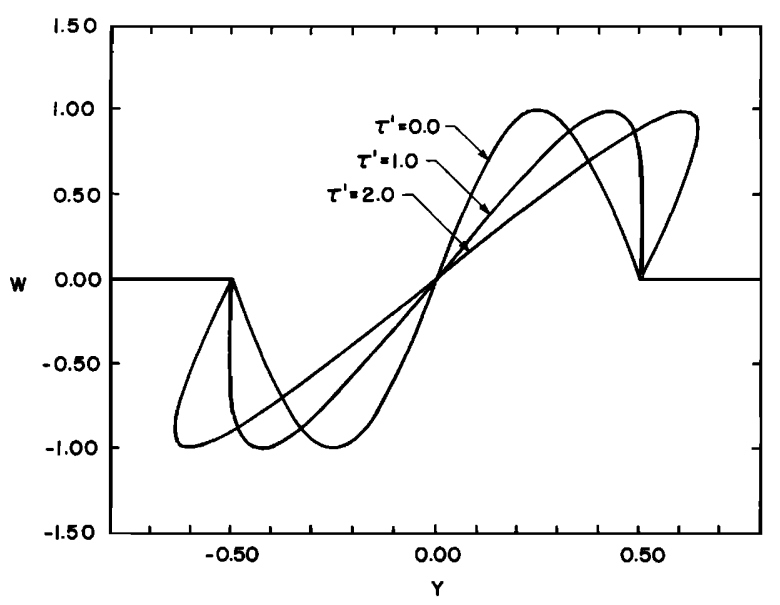

FIG. 13. The wave evolution of a shear sine pulse in vulcanized natural rubber at $\gamma_{s}^{(0)}=3.40$ prestrain $(\Gamma=0.1790)$. note that the results for solid polyurethane rubber are obtained when $v=0.5$ and $\beta=1$. At this limiting case, the constitutive relations are identical in form to the well-known Mooney-Rivlin model. By substituting the free-energy function (58) in (6), it is found that the scalar coefficients $\alpha_{0}$, $\alpha_{1}$, and $\alpha_{2}$ can be written

$$
\begin{aligned}
\alpha_{0}= & \mu_{0}\left[-\beta \mathrm{III}_{B}^{[-v /(1-2 v)-1 / 2]}\right. \\
& \left.+(1-\beta) \mathrm{III}_{B}^{[v /(1-2 v)-1 / 2\}}\right], \\
\alpha_{1}= & \mu_{0} \beta / \mathrm{III}_{B}^{1 / 2}+\left(\mu_{0} \beta / \mathrm{III}_{B}^{3 / 2}\right)(1-\beta), \\
\alpha_{2}= & -\mu_{0}(1-\beta) / \mathrm{III}_{B}^{3 / 2} .
\end{aligned}
$$

If the material constants are taken to be $\mu=22063 \mathrm{~Pa}$, $v=0.25$, and $\beta=0$, predictions based on (58) and (59) are in excellent agreement with the experimental results up to failure of the material. Blatz and Ko (1962) have shown that this occurs at a principal stretch of approximately 2.0. For strains larger than this value, no statement can be made regarding the validity or invalidity of this model with respect to foamed polyurethane rubber.

The simplifications presented for incompressible materials are not applicable to shear wave evolution in compressible materials. In particular, the momentum equations do not decouple as they do for the incompressible system. Equation (28) must be used for the calculation of the nondimensional wave speed $\lambda_{s}$, Eq. (31) must be used for calculation of $\Gamma$, and Eq. (40) must be used for calculation of $\Lambda$. Therefore, the wave evolution can not be inferred from a plot of Cauchy shear stress versus linear shear strain as could be done for an incompressible material. That is, knowledge of the quantities $\sigma_{5,55}$ and $\sigma_{5,555}$ is not sufficient to predict the signs of the nonlinearity parameters. In fact, the Blatz-Ko model of foamed polyurethane rubber results in $\sigma_{5,555}=0$ which would yield $\Lambda=0$ if equation (45) were employed.

A plot of $\Gamma$ and $\lambda_{s}^{2}$ versus shear prestrain is presented in Fig. 14. As in the incompressible material described in the previous section, $\Gamma$ is found to be negative for low prestrain values and the wave exhibits backward steepening behavior. Additionally, at the critical point $\gamma_{5}^{(0)}=0, \Lambda \approx-3.0$ which

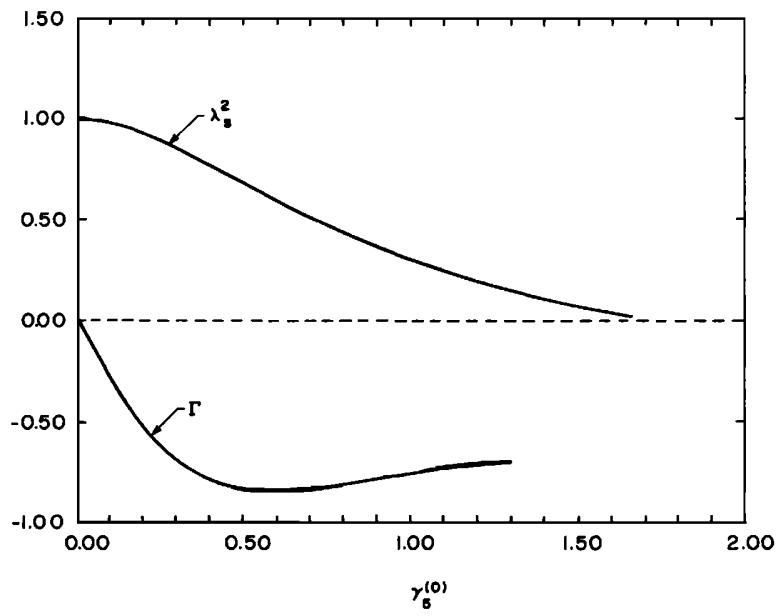

FIG. 14. Shear wave speed $\left(\lambda_{s}\right)^{2}$ and $\Gamma$ versus shear prestrain for the BlatzKo model of foamed polyurethane rubber. 
is of the same sign found for the incompressible case. Thus the wave evolution will be qualitatively similar to that seen in the incompressible case. A point of considerable interest in regards to Fig. 15 is that the square of the wave speed becomes negative at a value of $\gamma_{s}^{(0)}=1.6$, corresponding to a principal stretch of 2.08. As pointed out by Knowles and Sternberg (1975), this anomaly is associated with a loss of ellipticity of the statics equations. The value of the material constants, i.e., $\mu, \gamma$, and $\beta$, chosen for the constitutive relations describing the foamed polyurethane rubber are not valid at these large prestrain values because the specimen failed prior to this strain state.

In the range where it has been validated by experiments, the Blatz-Ko model for foamed polyurethane rubber does not predict a second critical point. However, one can find a range of material constants $\mu, v$, and $\beta$ such that $\gamma_{5}^{(0)} \neq 0$, and $\Gamma=0$ with $\left(\lambda_{s}\right)^{2}>0$. Values of $\left(\lambda_{s}\right)^{2}$ and $\Gamma$ have been computed and plotted in Fig. 15 for $\mu=22063 \mathrm{~Pa}, v=0.25$, and $\beta=0.5$. These computations reveal that $\Gamma=0$ at $\gamma_{s}^{(0)} \approx 3.7$ with a corresponding real wave speed. It therefore appears that the Blatz-Ko model is formally able to predict a second critical point. The authors are not aware of a specific material which is precisely modeled by these material constants. However, they have been chosen within the general realm of experimental parameters provided by Blatz and Ko (1962). It should be noted that these investigators indicated that $\beta$ appeared to be functionally dependent on the percent foam inherent in polyurethane rubber. In particular, as the percent of foam in the material decreased, $\beta$ increased and for the extreme case of $\beta=1$ the model was representative of solid polyurethane rubber. Therefore, the value $\beta=0.5$ chosen in the present example would be expected to be characteristic of a vulcanized natural rubber containing foam. It should also be noted that while the strain value $\left(\gamma_{s}^{(0)}=3.7\right.$ or principal stretch of 3.95 ) corresponding to the critical point is larger than the measured failure strain of foamed polyurethane rubber, these values are significantly lower than the strains achieved by the natural rubber represented in Fig. 3.

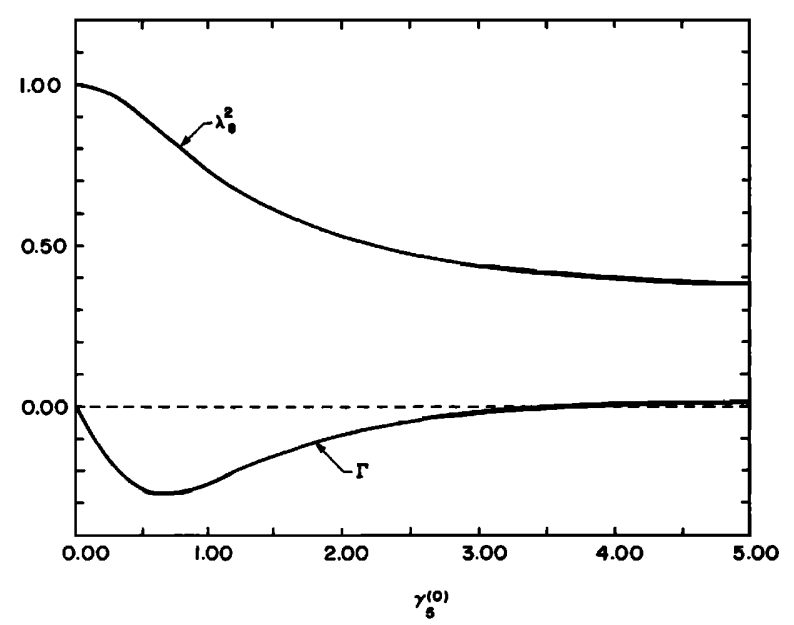

FIG. 15. Shear wave speed $\left(\lambda_{s}\right)^{2}$ and $\Gamma$ versus shear prestrain for the BlatzKo model of hypothetical polyurethane rubber when $\beta=0.5$.
In summary, the Blatz-Ko model admits the existence of a second critical point for what appears to be reasonable choices of $\mu, v$, and $\beta$. These results suggest that compressible materials may also possess a second critical point, however, the calculations presented here should not be regarded as a definitive demonstration.

\section{SUMMARY}

The propagation of a one-dimensional shear wave in a hyperelastic solid has been analyzed. The general expressions for the wave speed, the quadratic steepening parameter, and the cubic steepening parameter for both compressible and incompressible solids has been presented. The results of interest for incompressible materials are given by (24), (35) and (45) once $U=U\left(\gamma_{5}\right)$ is known. If the material is compressible, the appropriate free energy $U=U\left(I_{B}, I I_{B}, I I I_{B}\right)$ must be combined with (6), (7), and (12). The calculated stress gradients may then be combined with (19), (28), (31), (32), and (40)-(43) to compute the various speed and nonlinearity parameters.

For most prestrained states, the nonlinearity is quadratic and the evolution is therefore governed by (26) or (49). Near critical points, i.e., undisturbed states having $\Gamma=0$, higher-order corrections are required. As a result, either (36) or (50) yield the correct lowest-order solution. The latter case is seen to contain the results of the unstrained theory presented by previous investigators.

Numerical examples of an incompressible natural rubber revealed that a second critical point exists within the realm of experimentally attainable strains. The value of $\Lambda$ at this critical prestrain was noted to be different in sign than that found at the unstrained critical point, thus providing further evidence that the wave evolution and subsequent shock formation are highly dependent upon the prestrained state.

In Sec. VI, the Blatz-Ko model was employed to generate specific numerical results for compressible materials. The effect of prestraining foamed polyurethane was seen to produce a negative quadratic nonlinearity parameter. In spite of the significant differences in the complexity of the compressible equations, this result is seen to be similar to that found for incompressible materials.

The present study has also demonstrated that the BlatzKo model admits a second critical point at nonzero prestrain for reasonable choices of the Blatz-Ko material parameters $\mu, v$, and $\beta$. This second critical point is admissible in the sense that the linear wave speed is real. However, the numerical values of the material parameters are not associated with any specific material and it is felt that the identification of a second critical point in compressible materials remains as a task for future studies.

\section{ACKNOWLEDGMENTS}

This work was completed while the first author was supported by the Virginia Institute of Material Science and the second author was supported by the National Science Foundation under Grant No. CTS-891398. The authors would also like to acknowledge a number of useful conversations 
with Professor J. Jenkins and Professor P. Rosakis of Cornell University. These interactions have led to a number of improvements in the details as well as overall scope of the manuscript.

\section{APPENDIX: CONVERSION OF WAVE SPEEDS TO EULERIAN COORDINATES}

All results presented in the main text have been derived in terms of the Lagrangian coordinates introduced in Sec. $\mathbf{I}$. In order to relate our results more directly to experiments, it is natural to ask whether these have the same form in an Eulerian system. In the remainder of this Appendix, we convert the wave speeds corresponding to (26) and (36) to their Eulerian counterparts.

The dimensional versions of the nonlinear wave speed have already been given in (46) and (47). For the present purposes, it will be convenient to write these speeds as

$$
\frac{d X_{1}}{d t}=C_{s}\left(\lambda_{s}+\Gamma\left(\gamma_{5}-\gamma_{5}^{(0)}\right)+\frac{\Lambda}{2}\left(\gamma_{5}-\gamma_{5}^{(0)}\right)^{2}\right),
$$

where $X_{1}=X_{1}(t)$ is the equation of a characteristic line in the $X_{1}-t$ plane. Here, we simply recognize that the cubic term must be neglected when $\Gamma=O(1)$. The Eulerian wave speed may be derived by combining (9) with the equation of the characteristics $X_{1}=X_{1}(t)$. Differentiation of the first of (9) and use of implicit differentiation yields

$$
\begin{aligned}
\frac{d}{d t}\left[x_{1}(t)\right] & =\frac{d}{d t}\left\{X_{1}(t)+u_{1}\left[X_{1}(t), t\right]\right\} \\
& =\frac{d X_{1}}{d t}+\frac{\partial u_{1}}{\partial t}+\frac{\partial u_{1}}{\partial X_{1}} \frac{d X_{1}}{d t} .
\end{aligned}
$$

From the definition of $e_{1}$ and $v_{1}$ given in Sec. I, we find the the Eulerian version of the wave speed can be written

$$
\frac{d x_{1}}{d t}=\left(1+e_{1}\right) \frac{d X_{1}}{d t}+v_{1} .
$$

Use of (A1) yields

$$
\begin{aligned}
\frac{1}{C_{s}} \frac{d x_{1}}{d t}= & \lambda_{s}+\bar{v}_{1}+\lambda_{s} e_{1}+\left(1+e_{1}\right) \\
& \times\left[\Gamma\left(\gamma_{s}-\gamma_{s}^{(0)}\right)+(\Lambda / 2)\left(\gamma_{s}-\gamma_{s}^{(0)}\right)^{2}\right],
\end{aligned}
$$

where (18) has been employed. The presence of the $\bar{v}_{1}$ term is to be expected because the shear wave propagates relative to the material which is in turn set into motion in the propagation direction by the disturbance itself; the physical mechanism in this case is the nonlinear coupling to the longitudinal mode. That is, it represents the usual convection of the wave speed.

The result (A2) is exact within the context of (26) and (36). However, the terms involving $\Gamma$ and $\Lambda$ in (A2) are always small, regardless of whether the $\Gamma=O(1)$ or the $\Gamma=O(\epsilon)$ cases are considered, and (A2) may be approximated by

$$
\begin{aligned}
\frac{1}{C_{s}} \frac{d x_{1}}{d t} \approx & \lambda_{s}+\bar{v}_{1}+\lambda_{s} e_{1} \\
& +\Gamma\left(\gamma_{5}-\gamma_{5}^{(0)}\right)+\frac{\Lambda}{2}\left(\gamma_{5}-\gamma_{s}^{(0)}\right)^{2},
\end{aligned}
$$

with no loss in accuracy to the present theory. That is, the terms proportional to $e_{1}=O(1)$ will always be of the order of those already neglected in the present theory.

We now consider incompressible materials. In this case,

$$
\bar{v}_{1}=e_{1} \equiv 0
$$

and (A3) may then be written

$$
\frac{1}{C_{s}} \frac{d x_{1}}{d t}=\lambda_{s}+\Gamma\left(\gamma_{5}-\gamma_{s}^{(0)}\right)+\frac{\Lambda}{2}\left(\gamma_{5}-\gamma_{s}^{(0)}\right)^{2}
$$

with no further approximation. Thus, the Eulerian wave speed for incompressible materials is identical to the Lagrangian version (A1).

As already pointed out, shear waves in compressible materials generate nonzero values of $\bar{v}_{1}$ and $e_{1}$ due to nonlinear coupling. Both quantities are found to be of order $\epsilon$, i.e., of order of the shear wave amplitude, unless $\gamma_{s}^{(0)}=O(\epsilon)$. If we employ the original perturbation expansion (25), we find

$$
\begin{aligned}
\bar{v}_{1}+\lambda_{s} e_{1}= & \epsilon\left(w_{1}^{(1)}+\lambda_{s} w_{3}^{(1)}\right) \\
& +\epsilon^{2}\left(w_{1}^{(2)}+\lambda_{s} w_{3}^{(2)}\right)+O\left(\epsilon^{3}\right),
\end{aligned}
$$

where the fact that $w_{1}^{(0)}=w_{3}^{(0)}=0$ has been employed. The first term is obtained from the linear solution, i.e., the first of (25) combined with (29). When these formulas are employed, we find that

$$
w_{1}^{(1)}+\lambda_{s} w_{3}^{(1)}=r_{3} W\left(r_{1} / r_{3}+\lambda_{s}\right)=0,
$$

where (29) has been used to show that $r_{1}=-\lambda_{s} r_{3}$. Thus, the expansion (A4) may be rewritten

$$
\bar{v}_{1}+\lambda_{s} e_{1}=\epsilon^{2}\left(w_{1}^{(2)}+\lambda_{s} w_{3}^{(2)}\right)+O\left(\epsilon^{3}\right) .
$$

We may therefore conclude that the appropriate approximation to (A3) for compressible materials with $\Gamma=O(1)$ is

$$
\frac{1}{C_{s}} \frac{d x_{1}}{d t} \approx \lambda_{s}+\Gamma\left(\gamma_{5}-\gamma_{s}^{(0)}\right)+O\left(\epsilon^{2}\right),
$$

which is again equivalent to the Lagrangian version (46).

Finally, we consider the case of compressible materials with $\Gamma=O(\epsilon)$. In this case, we also need to solve the higher order equations for the first correction, i.e., $w_{\eta}^{(2)}$, to the linear approximation. If we combine (17) with (15), we find that the $\xi=3$ equation may be written

$$
\frac{\partial w_{3}}{\partial \bar{t}}-\frac{\partial}{\partial Y}\left(w_{1}+\lambda_{s} w_{3}\right)=0,
$$

exactly. Here, the dependent variables have been transformed from $X_{1}, \bar{t}$ to $Y=\bar{X}_{1}-\lambda_{s} \bar{t}, \bar{t}$. If we now note that the time variations are of order $\epsilon^{-2}$ when $\Gamma=O(\epsilon)$ and the fact that $w_{3}=O(\epsilon)$, at most, we find that

$$
\frac{\partial}{\partial Y}\left(w_{1}+\lambda_{s} w_{3}\right)=O\left(\epsilon^{3}\right) \text {. }
$$

Thus, if we assume $w_{1}+\lambda_{s} w_{3} \rightarrow 0$ as $Y \rightarrow \pm \infty$, we may recover the linear result (A5) as well as the corresponding result for the nonlinear correction: 


$$
w_{1}^{(2)}+\lambda_{s} w_{3}^{(2)}=0 \text {. }
$$

From (A3), (A6), and (A7), we conclude that the nonlinear Eulerian wave speed can be approximated by

$\frac{1}{C_{s}} \frac{d x_{1}}{d t} \approx \lambda_{s}+\Gamma\left(\gamma_{5}-\gamma_{5}^{(0)}\right)+\frac{\Lambda}{2}\left(\gamma_{5}-\gamma_{5}^{(0)}\right)^{2}+O\left(\epsilon^{3}\right)$, when $\Gamma=O(\epsilon)$.

In summary, we have shown that the Eulerian version of the nonlinear wave speed is identical to the Lagrangian version derived here, at least to the accuracy of the derived results.

Bains, J. A., and Breazeale, M. A. (1975). "Nonlinear Distortions of Ultrasonic Waves in Solids: Approach of a Stable Backward Sawtooth," J. Acoust. Soc. Am. 57, 745-746.

Blatz, P. J., and Ko, W. L. (1962). "Application of Finite Elastic Theory to the Deformation of Rubbery Materials," Trans. Soc. Rheolol. 6, 223251.

Carman, G. P., and Cramer, M. S. (1991). "Nonlinear Waves in Elastic Solids," VPI \& SU Eng. Rep. VPI-E-91-18.

Chu, B.-T. (1964). "Finite Amplitude Waves in Incompressible Perfectly Elastic Materials," J. Mech. Phys. Solids 12, 45-57.

Cramer, M. S., and Kluwick, A. (1984). "On the propagation of waves exhibiting both positive and negative nonlinearity," J. Fluid Mech. 169, 323-336.

Cramer, M. S., and Sen, R. (1990). "Mixed nonlinearity and double shocks in superfluid helium," J. Fluid Mech. 221, 233-261.

Cramer, M. S., and Sen, R. (1991). "A General Scheme for the Derivation of Evolution Equations Describing Mixed Nonlinearity," to appear in the J. Wave Motion.

Davison, L. (1966). "Propagation of Plane Waves of Finite Amplitude in Elastic Solids,” J. Mech. Phys. Solids 14, 249-270.
Garrett, S. (1981). "Nonlinear Distortion of 4th Sound in Superfluid ${ }^{3}$ He-B," J. Acoust. Soc. Am. 69, 139-144.

Hunter, S. C. (1983). Mechanics of Continuous Media (Halsted, New York) 2nd ed.

Knowles, J. K., and Sternberg, E. (1975). "On the Ellipticity of the Equations of Nonlinear Elastostatics for a Special Material," J. Elasticity 5, 341-361.

Lee-Bapty, I. P. (1981). "Nonlinear Wave Propagation in Stratified and Viscoelastic Media," Ph.D. dissertation, Leeds University, England.

Lee-Bapty, I. P., and Crighton, D. G. (1987). "Nonlinear Wave Motion Governed by the Modified Burgers' Equation," Philos. Trans. R. Soc. London Ser. A 323, 173-209.

Morris, F. E., and Nariboli, G. A. (1972). "Photoelastic waves," Int. J. Eng. Sci 10, 765-774.

Nariboli, G. A., and Lin, W. C. (1973). "A New Type of Burgers' Equation," ZAMM 53, 505-510.

Ogden, R. W. (1972). "Large Deformation Isotropic Elasticity-on the Correlation of Theory and Experiment for Incompressible Rubberlike Solids," Proc. R. Soc. London Ser. A 326, 565-584.

Peters, R. D., and Breazeale, M. A. (1968). "Third Harmonic of a Initially Sinusoidal Ultrasonic Wave in Copper," J. Appl. Phys. Lett. 12, 106.

Powell, R. L., and Schwarz, W. H. (1979). "Nonlinear Dynamic Viscoelasticity," J. Rheolol. 23 (3), 323-352.

Putterman, S. (1974). Superfluid Hydrodynamics (Elsevier, New York).

Taniuti, T., and Wei, C. C. (1968). "Reductive Perturbation Method in Nonlinear Wave Propagation," J. Phys. Soc. Jpn. 24, 941-946.

Thompson, R. B., Buck, O., and Thompson, D. O. (1976). "Higher Harmonics of Finite Amplitude Ultrasonic Waves in Solids," J. Acoust. Soc. Am. 59, 1087.

Thompson, R. B., and Tiersten, H. F. (1977). "Harmonic Generations of Longitudinal Elastic Waves,” J. Acoust. Soc. Am. 62, 37.

Thurston, R. N., and Shapiro, M. J. (1967). "Interpretations of Ultrasonic Experiments on Finite-Amplitude Waves,” J. Acoust. Soc. Am. 41, 1112.

Treloar, L. R. G. (1975). The Physics of Rubber Elasticity (Claredon, Oxford), 3rd ed. 